\title{
Characteristics of brain functional and structural connectivity in alexithymic students
}

This article was published in the following Dove Press journal:

Neuropsychiatric Disease and Treatment

\author{
Yantong Fang' \\ Mei $\mathrm{Li}^{2}$ \\ Minjun $\mathrm{Mei}^{2}$ \\ Xiaofei Sun ${ }^{2}$ \\ Dai Han ${ }^{1,3,4}$ \\ 'Children and Adolescents Mental \\ Health Joint Clinic, The Affiliated \\ Hospital of Hangzhou Normal \\ University, Hangzhou, Zhejiang, \\ People's Republic of China; ${ }^{2}$ Mental \\ Health Education and Counseling \\ Center, Hangzhou Normal University, \\ Hangzhou, Zhejiang, People's Republic \\ of China; Institutes of Psychological \\ Sciences, Hangzhou Normal \\ University, Hangzhou, Zhejiang, \\ People's Republic of China; ${ }^{4}$ Zhejiang \\ Key Laboratory for Research in \\ Assessment of Cognitive Impairments, \\ Hangzhou, Zhejiang, People's Republic \\ of China
}

Background: Alexithymia is a multifaceted personality trait and a risk factor for several mental and physical diseases.

Methods: In this study, 21 alexithymic students and 21 nonalexithymic students were recruited from the local university and assigned to the alexithymic group and the control group, respectively. Then, the functional connectivity and the structural connectivity among the brain regions of the students were investigated using resting-state functional magnetic resonance imaging (rsfMRI), function connection (FC) analysis, and diffusion tensor imaging (DTI).

Results: The rsfMRI results revealed 14 brain regions showing significant differences in the amplitude of low-frequency fluctuations between the two groups. Comparative analysis of the FC and DTI data in these brain regions between the two groups identified altered levels of functional and structural connectivity between the following four pairs of regions in the alexithymic subjects: the right inferior temporal gyrus and the central posterior gyrus, the left temporal gyrus and the insula, and the bilateral superior frontal gyrus and the anterior cingulate gyrus.

Conclusion: Compared with single MRI analysis, the multiple MRI analysis identified more precisely the brain regions that could play a key role in the development of alexithymia.

Keywords: rsfMRI, DTI, functional connectivity, structural connectivity, alexithymia

\section{Introduction}

Alexithymia is a personality trait characterized by difficulties in identifying and describing one's own emotional feelings and external thinking style. ${ }^{1}$ Several studies have confirmed the relationships between alexithymia and depression, anxiety, autism, and a number of other psychosomatic disorders. ${ }^{2-7}$ It has been reported that approximately $9 \%$ of people suffer from alexithymia. ${ }^{8}$ Clarifying the neural mechanism of alexithymia can improve the understanding about this personality trait and its relationships with psychosomatic disorders.

The development of functional magnetic resonance imaging (fMRI) in recent years has provided a precise and noninvasive technique for exploring the neuropathological basis of alexithymia. Previous studies using fMRI have suggested that alexithymia was associated with abnormal neural activity in several brain regions, including the prefrontal cortex, the cingulated cortex, the insula (INS), the amygdala, the precuneus, and the superior temporal gyrus. ${ }^{9-11}$ However, some of these regions are also known to be associated with several other mental disorders such as depression, anxiety, autism, and schizophrenia. ${ }^{12-15}$ Therefore, it is necessary to identify more precisely the brain regions that play a key role in the development of alexithymia. Furthermore, these studies have suggested that alexithymia was associated with multiple brain regions rather than only a single region. Multiple brain regions must cooperate with other
Correspondence: Dai Han Institutes of Psychological Science, Hangzhou Normal University, 2318 Yuhangtang Road, Hangzhou, Zhejiang Province 3I I I I, People's Republic of China

Email handai@hznu.edu.cn 
brain regions to maintain regular brain functions. Therefore, it is reasonable to hypothesize that the development of alexithymia is associated with not only the abnormal neural activity of several brain regions but also the somewhat abnormal functional or structural connectivity among these brain regions.

Function connection (FC) analysis provides a powerful method for investigating the functional connectivity among brain regions. Liemburg et $\mathrm{al}^{16}$ reported abnormal FC levels among the medial prefrontal cortex (mPFC), the occipital areas, the INS, and the anterior cingulate cortex (ACC) in individuals with alexithymia. In addition, structural connectivity is an important index that can be used to investigate the cooperative effects among the brain regions associated with alexithymia. Diffusion tensor imaging (DTI) is a highly sensitive method for measuring the structural integrality of the white matter tracts that connect the brain regions. A study using DTI conducted by Ho et $\mathrm{al}^{17}$ suggested that the superior longitudinal fasciculus, a primary white matter tract that connects the frontal, occipital, parietal, and temporal lobes, showed a significant correlation with alexithymia. These studies have partly supported the abovementioned hypothesis. Unfortunately, the study conducted by Liemburg et al ${ }^{16}$ investigated only the brain regions in the default network, and that of Ho et $\mathrm{al}^{17}$ focused only on the corpus callosum and the superior longitudinal fasciculus.

To verify the abovementioned hypothesis and identify more precisely the key brain regions associated with alexithymia, the present study was conducted to investigate the functional and structural connectivity features between each brain region showing abnormal neural activity in subjects with alexithymia. First, we identified all the brain regions that exhibited abnormal neural activity in alexithymic individuals using resting-state fMRI (rsfMRI). Then, we examined the FCs between each of these regions and the other regions of the entire brain using FC analysis. Finally, we examined the structural features of the FCs showing abnormal levels in alexithymic individuals using DTI analysis. Holistic knowledge about the functional and structural connectivity of the brain regions associated with alexithymia will contribute toward an in-depth understanding of the neural nature of this multifaceted personality trait, ie, alexithymia.

\section{Methods}

\section{Subjects}

All participants were recruited from among college students who participated in a freshmen mental health examination in 2015. All subjects were right-handed and had no history of brain injuries, substance abuse, or mental disorders. Those with contraindications to MRI scans or had consumed any psychotropic medications within 6 months before this study were excluded. Eligible alexithymic participants were randomly chosen, telephonically contacted, and assigned to the alexithymic group (Alex group). The eligible nonalexithymic participants, who matched the alexithymic participants in terms of age and sex, were randomly chosen, telephonically contacted, and assigned to the control group (Cont group). A total of 21 participants in the Alex group (age $=18.33 \pm 1.06$ years, eleven males and ten females) and 21 participants in the Cont group (age $=18.62 \pm 1.07$ years, eleven males and ten females) were recruited in this study. The study protocol was approved by the Research Ethics Review Board of Hangzhou Normal University. All subjects provided written informed consent in accordance with the Declaration of Helsinki.

\section{Psychological measurements}

The situation of alexithymia, depression, and anxiety of these participants was accessed, respectively, using the Chinese vision Toronto Alexithymia Scale-20 (TAS-20), Self-Rating depression Scale (SDS), and Self-Rating Anxiety Scale (SAS). TAS-20 was used to assess the status of alexithymia. TAS-20 has 20 entries, with scores of $1-5$ corresponding to "highly disagree" to "highly agree", and the score of the scale was the sum of the scores of each entry. The subjects with a TAS-20 score $>60$ were assigned to the Alex group, and those with a score $<52$ were included in the Cont group. ${ }^{18}$ SDS and SAS both include 20 items. The standardized score is obtained by the integral part of 1.25 -fold of the total score of 20 items. Subjective depression was defined as a standard SDS score $\geq 50$. Subjective anxiety was defined as a SAS score $\geq 53$. Therefore, all data of the participants with SDS score $\geq 50$ or SAS score $\geq 53$ should be excluded.

\section{MRI measurements}

All MRI scan processes were performed with a 3T whole-body MR imager (GE MR750, GE Healthcare, Chicago, IL, USA). The rsfMRI were acquired: sequence $=$ gradient recalled echo-echo planar images (GRE-EPI), axial slices, 43 slices, repetition time $(\mathrm{TR}) /$ echo time $(\mathrm{TE})=2,000 / 30 \mathrm{~ms}$, field of view $($ FOV $)=220 \times 220 \mathrm{~mm}$, resolution $=64 \times 64$, flip angle (FA) $=90^{\circ}$, total scan time $=480$ seconds. Participants were instructed to simply rest with their eyes closed, not to think of anything in particular, and not to fall asleep. The 3D T1-weighted images were acquired: sagittal slices, 180 slices, $\mathrm{TR} / \mathrm{TE}=8,100 / 3$, resolution $=256 \times 256$, 
FOV $=256 \times 256$, thickness $/$ gap $=1 / 0 \mathrm{~mm}, \mathrm{FA}=8^{\circ}$, total scan time $=305$ seconds. Lastly, DTI was acquired: Spin-Echo Echo-Planar Imaging sequence, 67 axial slices, TR/TE =8,600/ minimum, FOV $=192 \times 192$, matrix $=128 \times 128$, num shots $=1$, slice thickness $=1.5 \mathrm{~mm}$. Diffusion direction: TENSOR, 30 directions, $b$-value $=1,000 \mathrm{~s} / \mathrm{mm}^{2}$, NEX (number of excitation) $=2$, number of $\mathrm{T} 2$ images $=4$, voxel size $=1.5 \times 1.5 \times 1.5 \mathrm{~mm}^{3}$, total scan time $=593$ seconds.

\section{Data processing and statistical analysis}

Based on the MatLab 2011b (MathWorks, Natick, MA, USA), data preprocessing was performed using Statistical Parametric Mapping (SPM8, http://www.fil.ion.ucl.ac.uk/ spm/), the Data Processing Assistant for Resting-State fMRI (DPARSF, http://www.restfmri.net), and the rsfMRI data analysis toolkit (REST, http://www.restfmri.net). ${ }^{19,20}$ The images were corrected for slice-time differences, realigned for head motion correction, coregistered to T1, and spatially smoothed, according to a previous study's suggestion. ${ }^{21}$ The amplitude of low-frequency fluctuation (ALFF) map was calculated. The ALFF values of the whole brain gray matter regions of the subjects in the Alex group and the Cont group were voxel-by-voxel-based compared using the independent $t$-test of REST software to obtain the brain regions with significant differences $(P<0.01$, AlphaSim correction, cluster size $>21)$. The result was displayed using xjView software (http://www.alivelearn.net/xjview/).

The brain regions showing a significant difference on ALFF were selected as the region of interest (ROI) (diameter $=5 \mathrm{~mm}$ ), with the Montreal Neurological Institute coordinates shown in Table 1. The time series correlation coefficient between each ROI and each voxel in the whole brain was calculated by REST. After Fisher's z-transformation, the FC map of each ROI and the whole brain was obtained. The FC values of each ROI and the entire brain of the subjects in the Alex group and the Cont group were compared using the independent $t$-test of REST software to obtain the pairs of brain regions with significantly different $\mathrm{FC}$ values $(P<0.01$, AlphaSim correction, cluster size $>21))^{22,23}$ The result was visualized with the BrainNet Viewer (https:// www.nitrc.org/projects/bnv/). ${ }^{24}$ For each individual dataset, the FC values of these pairs of brain regions were extracted using REST. The FC values of the two groups were compared using Independent-Samples $t$-test by SPSS statistical software version 19.0 (IBM Corporation, Armonk, NY, USA).

The DTI data were processed using the Pipeline for Analyzing Brain Diffusion Images (PANDA, http://www.nitrc.org/ projects/panda/) and DTI Studio (https://www.mristudio.org/).
Table I Brain regions showing significant differences on ALFF between the Alex group and the Cont group

\begin{tabular}{|c|c|c|c|c|c|}
\hline \multirow[t]{2}{*}{ Brain regions (BA) } & \multirow[t]{2}{*}{$\begin{array}{l}\text { Voxel } \\
\text { size }\end{array}$} & \multicolumn{3}{|c|}{$\begin{array}{l}\text { Peak MNI } \\
\text { coordinate }\end{array}$} & \multirow[t]{2}{*}{$\begin{array}{l}\text { Peak } \\
t \text { value }\end{array}$} \\
\hline & & $x$ & $Y$ & $\mathbf{Z}$ & \\
\hline \multicolumn{6}{|l|}{ Alex $<$ Cont } \\
\hline PreCG & 30 & -24 & -18 & 66 & -4.0067 \\
\hline $\mathrm{ACC}$ & 24 & 0 & 0 & 30 & -3.4135 \\
\hline PostCG & 43 & 54 & -12 & 21 & -4.4691 \\
\hline INS & 23 & -30 & -27 & 21 & -3.6573 \\
\hline Thalamus, left & 92 & -15 & -24 & 15 & -4.4737 \\
\hline Primary visual cortex (VI) & 24 & 24 & -69 & 12 & -3.7334 \\
\hline HIP & 22 & -18 & -42 & 3 & -3.3616 \\
\hline Putamen, left & 60 & -30 & 0 & -3 & -4.1590 \\
\hline Putamen, right & 52 & 30 & -6 & -3 & -4.0324 \\
\hline \multicolumn{6}{|l|}{ Alex $>$ Cont } \\
\hline SFG_R & 21 & 18 & 66 & 6 & 3.6904 \\
\hline SFG_L & 37 & -21 & 60 & 0 & 4.0344 \\
\hline ITG_R & 66 & 63 & -48 & -9 & 4.7707 \\
\hline ITG_L & 22 & -57 & -63 & -12 & 4.0419 \\
\hline OFC_R & 21 & 45 & 48 & -15 & 3.4859 \\
\hline Parahippocampal gyrus & 99 & 24 & -30 & -36 & 3.8754 \\
\hline
\end{tabular}

Note: $P<0.01$, AlphaSim correction, cluster size $>21$.

Abbreviations: ACC, anterior cingulated cortex; Alex, alexithymia group; ALFF, amplitude of low-frequency fluctuations; Cont, control group; HIP, hippocampus; INS, insula; ITG_L, inferior temporal gyrus, left; ITG_R, inferior temporal gyrus, right; MNI, Montreal Neurological Institute; OFC_R, orbitofrontal cortex, right; PreCG, precentral gyrus; PostCG, postcentral gyrus; SFG_L, superior frontal gyrus, left; SFG_R, superior frontal gyrus, right.

The DTI data processing included transferring Digital Imaging and Communications in Medicine to Neuroimaging Informatics Technology Initiative, image registration, normalizing, and smoothing. Maps of FA were computed from the DTI data. Based on the FC analysis results, the FA values were extracted using the Fiber Assignment by Continuous Tracking algorithm. ${ }^{25}$ Data normality of FA in the two groups was assessed by the Kolmogorov-Smirnov test. Data showing a normal distribution were summarized by mean $\pm \mathrm{SD}$, and data showing a nonnormal distribution were summarized by median values. The FA values of the two groups were compared using Independent-Samples $t$-test by SPSS. Differences were considered to be significant at a two-sided $P$-value of 0.05 . The result was visualized by BrainNet Viewer.

\section{Results}

\section{Comparative analysis of ALFF between the two groups}

The results of the amplitude of ALFF analysis showed that the neural activities of nine brain regions were significantly lower, and the neural activities of six brain regions were significantly higher in the Alex group than those in the Cont group (Table 1, Figure 1). 


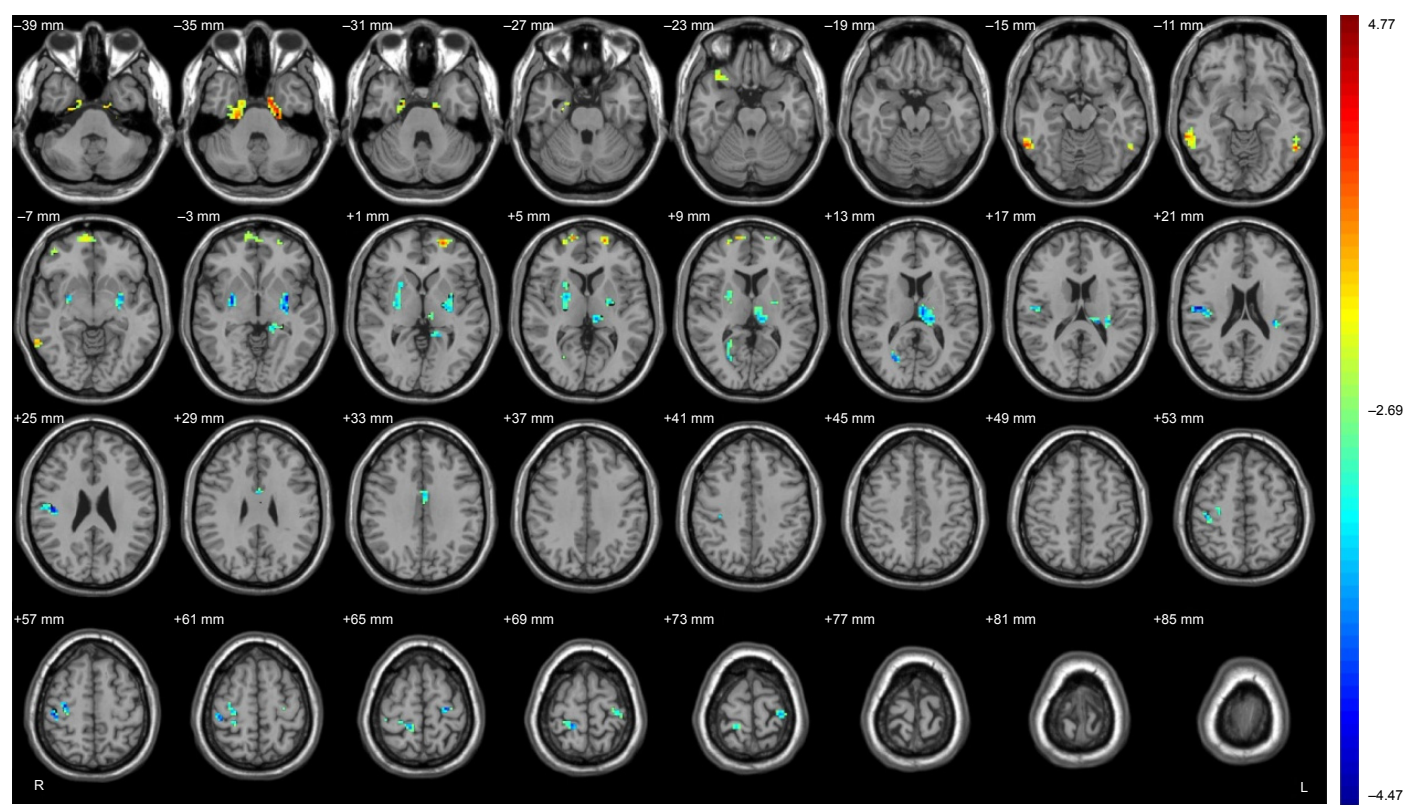

Figure I Brain regions showing significant differences on ALFF between Alex and Cont group.

Notes: $P<0.01$, AlphaSim correction, cluster size $>21$; red and yellow color means Alex $>$ Cont; blue and green color means Alex $<$ Cont.

Abbreviations: Alex, alexithymia group; ALFF, amplitude of low-frequency fluctuations; Cont, control group.

\section{Comparative analysis of functional connectivity between two groups}

The results of the analysis showed significantly different FC values of 14 pairs of regions, including inferior temporal gyrus (right) (ITG_R) and postcentral gyrus (preCG), ITG_R and V1, ITG_R and Putamen (left), ITG_R and Putamen (right), ITG_L and INS, superior frontal gyrus (right) (SFG_R) and postcentral gyrus (postCG), SFG_R and INS, orbitofrontal cortex (right) (OFC_R) and Putamen (left), Putamen (right) and INS, ITG_L and SFG_L, SFG_R and ACC, SFG_L and ACC, OFC_R and PostCG, OFC_R and hippocampus in the Alex group, compared with those in the Cont group (Table 2, Figure 2).

\section{Comparison analysis of functional connectivity between two groups}

The results revealed significantly different FA values of four pairs of regions, including the ITG_R and PostCG, ITG_L and INS, SFG_R and ACC, and SFG_L and ACC in the Alex group, compared with those in the Cont group (Table 3, Figure 2).

\section{Discussion}

Based on previous studies, this was the first study to compare the neurological activity of each brain region of subjects with and without alexithymia in the resting state. The results showed an altered neural activity in 15 regions (Table 1) in
Alex group compared with that in Cont group. Previous neuroimaging studies have also demonstrated abnormal activities in these 15 brain regions in subjects with alexithymia. ${ }^{9,26,27}$ Therefore, using these brain regions as the ROIs, this study indicated that the FC levels between 14 pairs of brain regions (Table 2) in the Alex group were found to be significantly different from those in the Cont group. It was notable to observe that all the brain regions involved in the altered

Table 2 Fourteen pairs of regions showing significant differences on FC between the Alex group and the Cont group

\begin{tabular}{lllll}
\hline Brain regions & Alex & Cont & $\boldsymbol{t}$ value & P-value \\
\hline ITG_R-PostCG & $0.243 \pm 0.054$ & $0.625 \pm 0.06 \mathrm{I}$ & -4.527 & $<0.00 \mathrm{I}$ \\
ITG_R-VI & $0.038 \pm 0.016$ & $0.105 \pm 0.035$ & -5.09 & $<0.00 \mathrm{I}$ \\
ITG_R-Putamen_L & $0.024 \pm 0.01 \mathrm{I}$ & $0.12 \pm 0.04 \mathrm{I}$ & -4.06 & $0.00 \mathrm{I}$ \\
ITG_R-Putamen_R & $0.013 \pm 0.044$ & $0.119 \pm 0.042$ & $-3.42 \mathrm{I}$ & 0.002 \\
ITG_L-INS & $0.339 \pm 0.048$ & $0.72 \mathrm{I} \pm 0.04 \mathrm{I}$ & -3.416 & 0.002 \\
SFG_R-PostCG & $0.116 \pm 0.047$ & $0.206 \pm 0.035$ & -3.687 & $0.00 \mathrm{I}$ \\
SFG_R-INS & $0.283 \pm 0.044$ & $0.314 \pm 0.047$ & -3.824 & $0.00 \mathrm{I}$ \\
OFC_R-Putamen_L & $0.201 \pm 0.05 \mathrm{I}$ & $0.234 \pm 0.044$ & -3.177 & 0.002 \\
Putamen_R-INS & $0.53 \pm 0.073$ & $0.592 \pm 0.064$ & -3.568 & $0.00 \mathrm{I}$ \\
ITG_L-SFG_L & $0.465 \pm 0.047$ & $0.398 \pm 0.052$ & 4.46 & $<0.00 \mathrm{I}$ \\
SFG_R-ACC & $0.639 \pm 0.046$ & $0.34 I \pm 0.039$ & 3.499 & 0.002 \\
SFG_L-ACC & $0.524 \pm 0.050$ & $0.34 I \pm 0.044$ & $4.27 \mathrm{I}$ & $0.00 \mathrm{I}$ \\
OFC_R-PostCG & $0.07 \mathrm{I} \pm 0.06 \mathrm{I}$ & $0.12 \pm 0.04 \mathrm{I}$ & 4.022 & $0.00 \mathrm{I}$ \\
OFC_R-Hippocampus & $0.427 \pm 0.075$ & $0.337 \pm 0.065$ & 4.127 & $0.00 \mathrm{I}$ \\
\hline
\end{tabular}

Abbreviations: ACC, anterior cingulated cortex; Alex, alexithymia group; Cont, control group; ITG_L, inferior temporal gyrus, left; ITG_R, inferior temporal gyrus, right; OFC_R, orbitofrontal cortex, right; PreCG, precentral gyrus; PostCG, postcentral gyrus; SFG_L, superior frontal gyrus, left; SFG_R, superior frontal gyrus, right. 

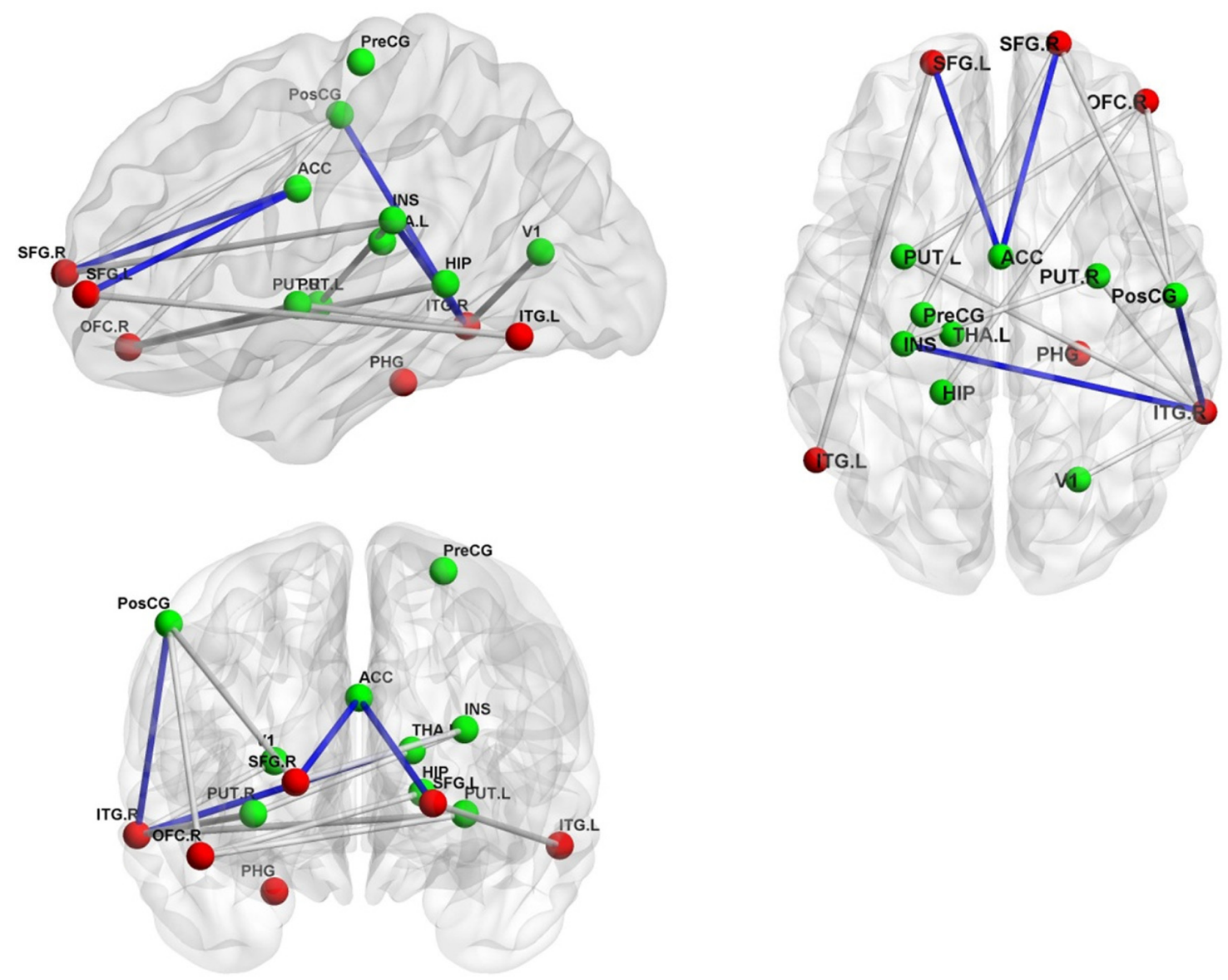

Figure 2 The pairs of regions showing the significant differences on FC or FA between the Alex group and the Cont group.

Notes: Red ball means Alex > Cont on ALFF; green ball means Alex < Cont on ALFF; gray line means the difference on FC between the Alex group and the Cont group is statistically significant; blue line means the difference on FA between the Alex group and the Cont group is statistically significant.

Abbreviations: ACC, anterior cingulate cortex; Alex, alexithymia group; ALFF, amplitude of low-frequency fluctuations; Cont, control group; HIP, hippocampus; INS, insula; ITG.L, inferior temporal gyrus, left; ITG.R, inferior temporal gyrus, right; PHG, parahippocampal gyrus; PreCG, precentral gyrus; PostCG, postcentral gyrus; PUT.L, PUTAMEN, left; PUT.R, Putamen, right; SFG.L, superior frontal gyrus, left; SFG.R, superior frontal gyrus, right; THA.L, thalamus_L; VI, primary visual cortex; OrbPFC.R, orbitofrontal cortex, right.

functional connectivity belonged to the 15 regions showing significant altered neural activity in this study. This implies that the functional connectivity results accurately identified all the brain regions that are actually associated with alexithymia. Finally, based on these 14 pairs of brain regions, the structural connectivity results demonstrated lower levels of structural connectivity between four pairs of regions (Table 3 )

Table 3 Brain regions showing significant differences on fractional anisotropy between the Alex group and the Cont group

\begin{tabular}{lllll}
\hline Brain regions & Alex group & Cont group & $\boldsymbol{t}$ value & $\boldsymbol{P}$-value \\
\hline ITG_R-PostCG & $0.362 \pm 0.0037$ & $0.378 \pm 0.0046$ & -2.386 & 0.022 \\
ITG_L-INS & $0.049 \pm 0.0034$ & $0.187 \pm 0.0053$ & $-2.18 \mathrm{I}$ & 0.035 \\
SFG_R-ACC & $0.189 \pm 0.0054$ & $0.369 \pm 0.0053$ & -2.688 & 0.010 \\
SFG_L-ACC & $0.517 \pm 0.0039$ & $0.536 \pm 0.0053$ & -2.933 & 0.006 \\
\hline
\end{tabular}

Abbreviations: ACC, anterior cingulate cortex; Alex, alexithymia group; Cont, control group; INS, insula; ITG_L, inferior temporal gyrus, left; ITG_R, inferior temporal gyrus, right; PostCG, postcentral gyrus SFG_L, superior frontal gyrus, left; SFG_R, superior frontal gyrus, right. in the Alex group. Both the functional connectivity and the structural connectivity of the four pairs of brain regions in the Alex group were significantly different from those in the Cont group, indicating that the uncooperative condition of the four pairs of brain regions could most probably be the neural basis underlying the development of alexithymia.

The human ITG is a part of the visual conduction pathway and is primarily responsible for visual processing. ${ }^{28}$ The PostCG is also known as the primary body sensory cortex and is responsible for receiving and organizing sensory information from the entire body. The INS has a wide range of fiber connectivity to other brain regions and is primarily responsible for body and visceral sensation, self-pain perception, visceral movement, and autonomic nervous control..$^{29,30}$ Pollatos and Gramann ${ }^{31}$ reported functional hyperactivity of ITG in a subject with hyperalexithymia. In addition, several studies have confirmed that the core symptom of 
alexithymia (blunted feeling) is related to the impaired function of the superior temporal gyrus. ${ }^{32,33}$ A few studies have also confirmed that alexithymia (difficulties identifying and describing one's own emotions) is associated with impaired interoception. ${ }^{34,35}$ Ernst et al $^{36}$ also demonstrated the association of interoceptive awareness and alexithymia with neurotransmitter concentrations in the INS and the ACC. ${ }^{36}$ Furthermore, a few clinical surveys and neuroimaging studies have suggested that alexithymia was associated with functional or structural impairments in the INS. ${ }^{37-39}$ Therefore, it is reasonable to suggest that the mechanism of alexithymia is associated with the dysfunction of the ITG, the PostCG, and the INS.

The SFG is primarily involved in the regulation of fine body movements and a variety of cognitive functions such as working memory, scenario memory, and attention. ${ }^{40,41}$ In addition, the SFG belongs to the default network and holds a wide range of fiber connectivity to other regions in the default network, such as the cingulate gyrus. ${ }^{42}$ The ACC is an important component of the cerebral limbic system and is primarily involved in several complex body and visceral motor functions and pain responses. ${ }^{43,44}$ Moreover, the ACC belongs to the default network and is responsible for the integration of various information inputs into the brain and the regulation of various complex cognitive processes by coordinating with other regions in the default network. ${ }^{43,45}$ Several previous studies have suggested the relationship between the dysfunctions of SFG and ACC and alexithymia. ${ }^{46-48}$ The present study has further demonstrated that the abnormal functional and structural connectivity between the bilateral SFG and ACC may be involved in the key mechanism underlying the development of alexithymia.

Interestingly, this study indicated the low-level structural connectivities between the four pairs of brain regions in the Alex group. The FC values between the bilateral ITG and the PostCG/INS were also lower in Alex group than those in the Cont group. However, the FC values between the bilateral SFG and ACC were higher in the Alex group than those in the Cont group. This inconsistency implies that a low-level structural connectivity induces a high- or low-level functional connectivity and that neither high-nor low-level FC presents a concordant or good functional connectivity.

\section{Conclusion}

This study analyzed the functional connectivity and the structural connectivity between the brain regions associated with alexithymia, primarily using rsfMRI, FC analysis, and DTI. Based on the data obtained from the multiple analysis methods, the abnormal functional and structural connectivity was precisely identified between the bilateral ITG and the PostCG/INS and between the bilateral SFG and ACC associated with alexithymia. A limitation of this study is the small sample size. To clarify the neural mechanism of alexithymia, more subjects will be recruited in a further study to delineate the relationship of alexithymia with the functional and structural connectivity between these four pairs of brain regions in the future.

\section{Acknowledgments}

This work was supported by a grant from Zhejiang Provincial Natural Science Foundation of China (No LQ14H090004) and the China Scholarship Council (201708330165).

\section{Author contributions}

Dai Han designed and managed the imaging data selection and analysis. Yantong Fang wrote the first draft of the manuscript. Yantong Fang, Mei Li, Minjun Mei, and Xiaofei Sun undertook the psychology measurement and statistical analysis. All authors contributed toward data analysis, drafting and critically revising the paper and agree to be accountable for all aspects of the work.

\section{Disclosure}

The authors report no conflicts of interest in this work.

\section{References}

1. Taylor GJ, Bagby RM, Parker JDA. What's in the name 'alexithymia'? A commentary on "Affective agnosia: expansion of the alexithymia construct and a new opportunity to integrate and extend Freud's legacy." Neurosci Biobehav Rev. 2016;68:1006-1020.

2. Kojima M. Alexithymia as a prognostic risk factor for health problems: a brief review of epidemiological studies. Biopsychosoc Med. 2012; 6(1):21-29.

3. Taylor GJ, Bagby RM, Parker JDA. Disorders of Affect Regulation: Alexithymia in Medical and Psychiatric Illness. Cambridge, UK: Cambridge University Press; 1997.

4. Han D, Zhang Y, Li B, et al. Alexithymia in Chinese chronic obstructive pulmonary disease (COPD) patients: the prevalence and related factors of alexithymia. Psychiatry Res. 2012;198(2):274-278.

5. Lumley MA, Neely LC, Burger AJ. The assessment of alexithymia in medical settings: implications for understanding and treating health problems. J Pers Assess. 2007;89(3):230-246.

6. Shah P, Hall R, Catmur C, Bird G, Alexithymia BG. Alexithymia, not autism, is associated with impaired interoception. Cortex. 2016;81: 215-220.

7. Tella MD, Enrici I, Castelli L, et al. Alexithymia, not fibromyalgia, predicts the attribution of pain to anger-related facial expressions. J Affect Disord. 2018;227:272-279.

8. Joukamaa M, Taanila A, Miettunen J, Karvonen JT, Koskinen M, Veijola J. Epidemiology of alexithymia among adolescents. J Psychosom Res. 2007;63(4):373-376.

9. Moriguchi Y, Komaki G. Neuroimaging studies of alexithymia: physical, affective, and social perspectives. Biopsychosoc Med. 2013;7(1):8-12.

10. Goerlich-Dobre KS, Bruce L, Martens S, Aleman A, Hooker CI. Distinct associations of insula and cingulate volume with the cognitive and affective dimensions of alexithymia. Neuropsychologia. 2014;53(4): 284-292. 
11. Ihme K, Sacher J, Lichev V, et al. Alexithymic features and the labeling of brief emotional facial expressions - An fMRI study. Neuropsychologia. 2014;64:289-299.

12. Yuan H, Young KD, Phillips R, Zotev V, Misaki M, Bodurka J. Restingstate functional connectivity modulation and sustained changes after real-time functional magnetic resonance imaging neurofeedback training in depression. Brain Connect. 2014;4(9):690-701.

13. Spielberg JM, Miller GA, Warren SL, Sutton BP, Banich M, Heller W. Transdiagnostic dimensions of anxiety and depression moderate motivation-related brain networks during goal maintenance. Depress Anxiety. 2014;31(10):805-813.

14. Ursu S, Kring AM, Gard MG, et al. Prefrontal cortical deficits and impaired cognition-emotion interactions in schizophrenia. Am J Psychiatry. 2011;168(3):276-285.

15. Phillips ML, Drevets WC, Rauch SL, Lane R. Neurobiology of emotion perception II: Implications for major psychiatric disorders. Biol Psychiatry. 2003;54(5):515-528.

16. Liemburg EJ, Swart M, Bruggeman R, et al. Altered resting state connectivity of the default mode network in alexithymia. Soc Cogn Affect Neurosci. 2012;7(6):660-666.

17. Ho Ns, Wong MM, Lee TM. Neural connectivity of alexithymia: Specific association with major depressive disorder. J Affect Disord. 2016;193:362-372.

18. Bagby RM, Parker JD, Taylor GJ. The twenty-item Toronto Alexithymia Scale - I. Item selection and cross-validation of the factor structure. J Psychosom Res. 1994;38(1):23-32.

19. Song XW, Dong ZY, Long XY, et al. REST: a toolkit for resting-state functional magnetic resonance imaging data processing. PLoS One. 2011;6(9):e25031.

20. Chao-Gan Y, Yu-Feng Z, Yan, Zang YF. DPARSF: A MATLAB Toolbox for "Pipeline" Data Analysis of Resting-State fMRI. Front Syst Neurosci. 2010;4:13.

21. Ran Q, Chen J, Li C, et al. Abnormal amplitude of low-frequency fluctuations associated with rapid-eye movement in chronic primary insomnia patients. Oncotarget. 2017;8(49):84877-84888.

22. Kühn S, Gallinat J. Brains online: structural and functional correlates of habitual Internet use. Addict Biol. 2015;20(2):415-422.

23. Shi Y, Zeng Y, Wu L, et al. A Study of the Brain Abnormalities of Post-Stroke Depression in Frontal Lobe Lesion. Sci Rep. 2017;7(1).

24. Xia M, Wang J, He Y, Yong H. BrainNet Viewer: A Network Visualization Tool for Human Brain Connectomics. PLoS One. 2013;8(7):e68910.

25. Jiang H, van Zijl PCM, Kim J, Pearlson GD, Mori S. DtiStudio: Resource program for diffusion tensor computation and fiber bundle tracking. Comput Methods Programs Biomed. 2006;81(2):106-116.

26. van der Velde J, Servaas MN, Goerlich KS, et al. Neural correlates of alexithymia: a meta-analysis of emotion processing studies. Neurosci Biobehav Rev. 2013;37(8):1774-1785.

27. Kano M, Fukudo S. The alexithymic brain: the neural pathways linking alexithymia to physical disorders. Biopsychosoc Med. 2013;7(1):1.

28. Haxby JV, Hoffman EA, Gobbini MI. The distributed human neural system for face perception. Trends Cogn Sci. 2000;4(6):223-233.

29. Oppenheimer SM, Gelb A, Girvin JP, Hachinski VC. Cardiovascular effects of human insular cortex stimulation. Neurology. 1992;42(9): 1727-1732.

30. Critchley HD. Neural mechanisms of autonomic, affective, and cognitive integration. J Comp Neurol. 2005;493(1):154-166.
31. Pollatos O, Gramann K. Attenuated modulation of brain activity accompanies emotion regulation deficits in alexithymia. Psychophysiology. 2012;49(5):651-658.

32. Goerlich-Dobre KS, Witteman J, SchillerNO, van Heuven VJP, Aleman A, Martens S. Blunted feelings: Alexithymia is associated with a diminished neural response to speech prosody. Soc Cogn Affect Neurosci. 2014;9(8):1108-1117.

33. Reker M, Ohrmann P, Rauch AV, et al. Individual differences in alexithymia and brain response to masked emotion faces. Cortex. 2010;46(5):658-667.

34. Murphy J, Brewer R, Hobson H, Catmur C, Bird G. Is alexithymia characterised by impaired interoception? Further evidence, the importance of control variables, and the problems with the Heartbeat Counting Task. Biol Psychol. 2018;136:189-197.

35. Sowden S, Brewer R, Catmur C, Bird G. The specificity of the link between alexithymia, interoception, and imitation. J Exp Psychol Hum Percept Perform. 2016;42(11):1687-1692.

36. Ernst J, Böker H, Hättenschwiler J, et al. The association of interoceptive awareness and alexithymia with neurotransmitter concentrations in insula and anterior cingulate. Soc Cogn Affect Neurosci. 2014;9(6): $857-863$.

37. Donges U-S, Suslow T. Alexithymia and automatic processing of emotional stimuli: a systematic review. Rev Neurosci. 2017;28(3):247.

38. Sutherland MT, Carroll AJ, Salmeron BJ, Ross TJ, Stein EA. Insula's functional connectivity with ventromedial prefrontal cortex mediates the impact of trait alexithymia on state tobacco craving. Psychopharmacology. 2013;228(1):143-155.

39. Hogeveen J, Bird G, Chau A, Krueger F, Grafman J. Acquired alexithymia following damage to the anterior insula. Neuropsychologia. 2016;82:142-148.

40. Nachev P, Kennard C, Husain M. Functional role of the supplementary and pre-supplementary motor areas. Nat Rev Neurosci. 2008;9(11): 856-869.

41. Levy R, Goldman-Rakic PS. Segregation of working memory functions within the dorsolateral prefrontal cortex. Exp Brain Res. 2000; 133(1):23-32.

42. Li W, Qin W, Liu H, et al. Subregions of the human superior frontal gyrus and their connections. Neuroimage. 2013;78:46-58.

43. Gasquoine PG. Localization of function in anterior cingulate cortex: from psychosurgery to functional neuroimaging. Neurosci Biobehav Rev. 2013;37(3):340-348.

44. Fuchs PN, Peng YB, Boyette-Davis JA, Uhelski ML. The anterior cingulate cortex and pain processing. Front Integr Neurosci. 2014;8:35.

45. Kucian K, von Aster M, Loenneker T, Dietrich T, Martin E. Development of neural networks for exact and approximate calculation: a FMRI study. Dev Neuropsychol. 2008;33(4):447-473.

46. Berthoz S, Artiges E, van de Moortele PF, et al. Effect of impaired recognition and expression of emotions on frontocingulate cortices: an fMRI study of men with alexithymia. Am J Psychiatry. 2002;159(6) 961-967.

47. Schäfer R, Popp K, Jörgens S, Lindenberg R, Franz M, Seitz RJ. Alexithymia-like disorder in right anterior cingulate infarction. Neurocase. 2007;13(3):201-208.

48. Gündel H, López-Sala A, Ceballos-Baumann AO, et al. Alexithymia correlates with the size of the right anterior cingulate. Psychosom Med. 2004;66(1):132-140.
Neuropsychiatric Disease and Treatment

\section{Publish your work in this journal}

Neuropsychiatric Disease and Treatment is an international, peerreviewed journal of clinical therapeutics and pharmacology focusing on concise rapid reporting of clinical or pre-clinical studies on a range of neuropsychiatric and neurological disorders. This journa is indexed on PubMed Central, the 'PsycINFO' database and CAS,

\section{Dovepress}

and is the official journal of The International Neuropsychiatric Association (INA). The manuscript management system is completely online and includes a very quick and fair peer-review system, which is all easy to use. Visit http://www.dovepress.com/testimonials.php to read real quotes from published authors. 